\title{
Analysis of Clinical Predictive Factors Affecting the Outcome of Second-Line Chemotherapy for Gemcitabine-Refractory Advanced Pancreatic Cancer
}

\author{
Jeung Eun Lee ${ }^{1}$, Hee Seung Lee ${ }^{2}$, Moon Jae Chung ${ }^{2}$, Jeong Youp Park ${ }^{2}$, Seung Woo Park ${ }^{2}$, Si Young Song ${ }^{2}$, and Seungmin \\ Bang $^{1}$ \\ ${ }^{\prime}$ Department of Internal Medicine and ${ }^{2}$ Institute of Gastroenterology, Yonsei University College of Medicine, Seoul, Korea
}

Background/Aims: The benefit of second-line chemotherapy (SL) after failed first-line chemotherapy (FL) in patients with advanced pancreatic cancer has not yet been established. We evaluated the clinical characteristics affecting the benefits of SL compared to best supportive care (BSC), identified the prognostic factors, and ultimately devised a model of clinical parameters to assist in making decision between SL and BSC after the failure of gemcitabine-based FL. Methods: The records of patients who received gemcitabinebased FL for advanced pancreatic cancer at Yonsei University Hospital between January 2010 and December 2015 were retrospectively reviewed. Significant clinical parameters were assessed for their potential as predictive factors. Results: SL patients received a longer duration of FL compared with BSC patients with median duration being 16.0 weeks (range, 8.0 to 26.0 weeks) and 8.0 weeks (range, 4.0 to 16.0 weeks), respectively $(p<0.001)$. When the SL group was stratified by their modified overall survival (mOS) (longer and shorter than 6 months), we found significant differences for several clinical factors, namely, metastasis to the peritoneum $(p<0.001)$, number of metastases $(p<0.001)$, thrombotic events $(p=0.003)$, and level of carbohydrate antigen 19-9 (CA199; $p=0.011$ ). In multivariate analysis, more than one site of metastasis, occurrence of thrombotic event during FL, and a CA19-9 level above $90 \mathrm{U} / \mathrm{mL}$ were significant independent prognostic factors for mOS in the SL group $(p<0.05)$. When an attempt was made to devise a prognostic nomogram, Harrell's C-index of the final prognosis prediction model was 0.62. Conclusions: SL may be beneficial for patients without peritoneal metastasis or thrombotic events who have a single metastasis and a level of CA19-9 less than $90 \mathrm{U} / \mathrm{mL}$. This prognostic nomogram can be used to predict mOS be- fore the administration of SL after the failure of gemcitabinebased FL. (Gut Liver 2020;14:135-143)

Key Words: Pancreatic neoplasms; Second line chemotherapy; Prognostic model

\section{INTRODUCTION}

Pancreatic cancer is a leading cause of cancer death worldwide with an estimated 5-year survival rate of 5\% to $6 \%{ }^{1,2}$ From 1990s, gemcitabine has been positioned as the backbone drug for the first-line chemotherapy (FL) in unresectable pancreatic cancer, proving the improvement of overall survival (OS). ${ }^{3}$ Despite the substantial development in palliative chemotherapies, the median OS is still less than 8 months with frequent resistance in FL. ${ }^{4,5}$ Numerous second-line chemotherapies (SLs) have been attempted to improve OSs of the patients enfacing the treatment failure of gemcitabine based FL. ${ }^{6}$ Currently, the CONKO-003 study presented the OFF regimen including oxaliplatin, folinic acid and 5-fluorouracil (5-FU), as an effective SL therapy after gemcitabine refractory treatment, demonstrating significant improvement in 0S. ${ }^{7}$ However, there are no other large randomized studies to establish the consensus of SL after disease progression of FL treatment. Furthermore, there is no evidence proving the superiority of SL with best supportive care (BSC) after failure of first line treatment failure.,

Our study was to analyze the clinical characteristics of patients who have received SL or discontinued salvage chemotherapy and went on with BSC after FL treatment. The primary endpoint was to evaluate clinical characteristics affecting the benefits from receiving SL comparing to BSC. The second endpoint of our study was to establish a prognostic scoring system

\footnotetext{
Correspondence to: Seungmin Bang

Department of Internal Medicine, Institute of Gastroenterology, Yonsei University College of Medicine, 50-1 Yonsei-ro, Seodaemun-gu, Seoul 03722, Korea

Tel: +82-2-2228-5221, Fax: +82-2-361-2125, E-mail: bang7028@yuhs.ac

Received on September 12, 2018. Revised on February 3, 2019. Accepted on February 7, 2019. Published online November 14, 2019. pISSN 1976-2283 eISSN 2005-1212 https://doi.org/10.5009/gnl18419

(a) This is an Open Access article distributed under the terms of the Creative Commons Attribution Non-Commercial License (http://creativecommons.org/licenses/by-nc/4.0) which permits unrestricted non-commercial use, distribution, and reproduction in any medium, provided the original work is properly cited.
} 
for an optimal decision of proceeding SL after the failure of gemcitabine-based FL.

\section{MATERIALS AND METHODS}

All patients with histologically proven pancreatic adenocarcinoma treated at Gastroenterology Unit of Yonsei University Hospital, Seoul, Republic of Korea, between January 2010 and December 2015 were involved in the development cohort. Patients were considered eligible for medical evaluation of SL indication if they had received one previous line of gemcitabinebased FL, including gemcitabine-based adjuvant chemotherapy in patients who had undergone curative resection. The external validation cohort included patients with proven pancreatic adenocarcinoma who received gemcitabine-based FL in the same institution as the development cohort between January 2016 and December 2016. All clinical, biological, and radiological parameters of the data were measured at the beginning of SL or at the end of FL for patients who proceeded with BSC. Treatment outcomes were retrospectively collected from medical records.

This is a retrospective study that only involves the collection of existing clinical data and medical records. The research did not include any direct or indirect identification information of study participants. The study participants' privacy, confidentiality and anonymity were guaranteed. The rights or interests of study participants were not violated. The risk of the study participant was not greater than the minimum risk. Because of the retrospective nature of the study, the informed consent of each participant was not available. According to the guidelines for the review of major ethical issues in Republic of Korea's clinical research, this type of study meets the conditions of exemption from formal ethics approval and written informed consent.

OS was calculated from the date of initiation of FL to the date of death from any cause. Modified OS (mOS) was calculated from the date of first administration of SL to the date of death from any cause. For patients who only received FL and went on with BSC after progression, mOS was measured from the end of FL to death of any cause. For patients with history of curative operation following cancer progression after gemcitabine-based adjuvant chemotherapy, mOS was measured from the initiating date of first line palliative chemotherapy to the date of death from any cause.

Median value (interquartile range) and frequency (percentage) were provided for the description of continuous and categorical variables, respectively. Medians and proportions were compared using the Student t-test and chi-square test (or Fisher exact test, if appropriate), respectively. The Cox proportional hazard models were performed to estimate hazard ratio and 95\% confidence interval (CI) for prognostic factors associated with mOS. The association of baseline parameters of mOS was first assessed by dividing the patient into two groups by mOS; less than 6 months and more than 6 months. Additionally, baseline param- eters of mOS was assessed by univariate Cox analyses, and then parameters with $\mathrm{p}$-values of less than 0.05 were entered into the final multivariable Cox regression model, after considering collinearity among variables with a correlation matrix.

Sensitivity analysis to explore the reliability of the final prognosis predictive model was performed with a stratified and a frailty approach by using a random component for the hazard function based on the regimen, and with a full-model and forward procedure. Accuracy of the final predictive model was verified regarding the discrimination parameter. The predictive value and the discrimination ability of the final model were assessed internally and externally with the Harrell's concordance index (C-index) and incremental area under the curve (iAUC).

The prognostic score was constructed with total points calculated by the nomogram. To identify risk groups and determine their survival benefit with SL, the Kaplan-Meier method with log-rank tests was used for comparisons between prognostic subgroups classified by the nomogram scoring.

All analyses were performed using SPSS version 23.0 (IBM Corp., Armonk, NY, USA) and R software version 2.15.2 (R Development Core Team, Vienna, Austria; http://www.r-project. org). Two-sided p-values less than 0.05 were considered statistically significant.

\section{RESULTS}

\section{Characteristics of SL group compared to BSC group after FL failure}

The median OS of SL patients versus BSC patients was 40 weeks (range, 28.0 to 56.0 weeks) versus 16.0 weeks (range, 12.0 to 32.0 weeks; $\mathrm{p}<0.001$ ). The median OS after the progression of FL of SL group (mOS) was 20.0 weeks (range, 12.0 to 32.0 weeks; $\mathrm{p}<0.001$ ). The median duration of BSC of FL only group after progression of FL (mOS) was 8.0 weeks (range, 4.0 to 16.0 weeks; $\mathrm{p}<0.001$ ).

There was significant difference in sex between two groups (evaluable in 501 patients; male: SL 47.3\% vs BSC 62.9\%; $\mathrm{p}=0.001$ ). The Eastern Cooperative Oncology Group (ECOG) performance status was significantly higher in SL patients at the initiation of palliative SL chemotherapy (evaluable in 501 patients; ECOG 0: SL 83.7\% vs BSC 61.7\%; p<0.001). There was a significant difference in primary tumor localization between two groups (evaluable in 501 patients; localization at head: SL 52.2\% vs BSC 38.3\%; $\mathrm{p}=0.002$ ).

A significantly higher number of SL patients received longer duration of FL (evaluable in 501 patients; SL group median 16.0 weeks [range, 8.0 to 26.0 weeks] vs BSC median 8.0 weeks [range, 4.0 to 16.0 week]; $\mathrm{p}<0.001)$. Significantly more patients in the SL group were diagnosed with metastasis to the lung (SL 20.4\% vs BSC 10.5\%; $p=0.003$ ) and peritoneum (SL 32.7\% vs BSC 40.8\%; $\mathrm{p}=0.001)$.

There were no differences detected between SL group and 
BSC group regarding age, metastatic lesion (liver, bone, distant lymph node and other sites including adrenal gland at spleen), number of metastasis after FL progression, preexisting diabetes, thrombosis event during FL and body mass index (BMI). Moreover, there was no significant difference in tumor markers carbohydrate antigen 19-9 (CA19-9) (Table 1).

\section{Characteristics of m0S above 6 months and moS below 6 months in SL group}

SL patients were divided into two groups depending on the mOS duration: mOS less than 6 months and greater than 6 months. The median mOS of the mOS less than 6 months group was 12.0 weeks (range, 8.0 to 24.0 weeks). The median m0S of the mOS greater than 6 months group was 36.0 weeks (range, 28.0 to 52.0 weeks).

In analysis of 245 patients with metastasis before initiation of SL, single metastasis was more common in the mOS greater than 6 months group (mOS less than 6 months group 42.0\% vs mOS greater than 6 months group; $\mathrm{p}=0.001$ ) and multiple metastasis was more common in the mOS less than 6 months group (mOS less than 6 months group 58.0\% vs mOS greater than 6 months group; $\mathrm{p}<0.001$ ).

Peritoneal metastasis was significantly more frequent in mOS below 6-month group (evaluable in 245 patients; peritoneal metastasis: mOS less than 6 months group 43.5\% vs mOS greater more than 6 months group $18.7 \% ; \mathrm{p}<0.001$ ).

Table 1. Baseline Characteristics of BSC Patients and SL Patients after FL Failure

\begin{tabular}{|c|c|c|c|}
\hline Characteristic & BSC $(n=256)$ & SL $(n=245)$ & $\mathrm{p}$-value \\
\hline Male sex & $161(62.9)$ & $116(47.3)$ & 0.001 \\
\hline Age, yr & $64.0(55.0-71.0)$ & $62.0(55.5-69.0)$ & 0.151 \\
\hline ECOG 0 & $158(61.7)$ & 205 (83.7) & $<0.001$ \\
\hline Tumor localization & & & 0.002 \\
\hline Head & 98 (38.3) & $128(52.2)$ & \\
\hline Body and tail & $142(55.5)$ & $98(40.0)$ & \\
\hline Overlapping & $16(6.3)$ & $19(7.8)$ & \\
\hline \multicolumn{4}{|l|}{ Metastatic lesion } \\
\hline Liver & $174(68.0)$ & $168(68.6)$ & 0.924 \\
\hline Lung & 27 (10.5) & $50(20.4)$ & 0.003 \\
\hline Bone & $21(8.2)$ & $11(4.5)$ & 0.102 \\
\hline Others & $24(9.4)$ & $18(7.3)$ & 0.426 \\
\hline Distant lymph node & 35 (13.7) & $50(20.4)$ & 0.056 \\
\hline Peritoneum & $123(40.8)$ & $80(32.7)$ & 0.001 \\
\hline No. of metastasis & & & 0.929 \\
\hline Single & $138(53.9)$ & $131(53.5)$ & \\
\hline Multiple & $118(46.1)$ & $114(46.5)$ & \\
\hline FL duration, wk & $8.0(4.0-16.0)$ & $16.0(8.0-26.0)$ & $<0.001$ \\
\hline OS & $16.0(12.0-32.0)$ & $40.0(28.0-56.0)$ & $<0.001$ \\
\hline mOS & $8.0(4.0-16.0)$ & $20.0(12.0-32.0)$ & $<0.001$ \\
\hline Diabetes mellitus & $81(31.6)$ & 95 (38.8) & 0.111 \\
\hline Thrombosis & $30(11.7)$ & $31(12.7)$ & 0.786 \\
\hline CA19-9 $\geq 90.0 \mathrm{U} / \mathrm{mL}$ & $193(75.4)$ & $157(64.1)$ & 0.006 \\
\hline BMI, $\mathrm{kg} / \mathrm{m}^{2}$ & $22.2(20.32-23.60)$ & $22.7(21.95-23.82)$ & 0.335 \\
\hline SL type & - & & - \\
\hline FOLFIRINOX & & $90(36.7)$ & \\
\hline FOLFOX & & 29 (11.8) & \\
\hline Capecitabine+oxaliplatin & & $98(40.0)$ & \\
\hline FEP & & 28 (11.4) & \\
\hline
\end{tabular}

Data are presented as number (\%) or median (interquartile range).

BSC, best supportive care; SL, second-line chemotherapy; FL, first-line chemotherapy; ECOG, Eastern Cooperative Oncology Group; OS, overall survival; mOS: modified OS; CA19-9, carbohydrate antigen 19-9; BMI, body mass index; FOLFIRINOX, 5-FU+folinic acid+irinotecan+oxaliplatin; FOLFOX, folinic acid+fluorouracil+oxaliplatin; FEP, folinic acid+etoposide+cisplatin. 
Thrombotic events were significantly more frequent in the mOS less than 6 months group (evaluable in 245 patients; thrombotic event: mOS less than 6 months group 18.1\% vs mOS greater than 6 months group 5.6\%; $p=0.003$ ).

CA19-9 measured after FL progression was significantly higher in the mOS less than 6 months group (evaluable in 245 patients; CA19-9 $\geq 90 \mathrm{U} / \mathrm{mL}$ : mOS less than 6 months group; $71.0 \%$ vs mOS greater than 6 months group; 55.1\%; $p=0.011$ ).

There were no significant differences detected between two groups regarding sex, age, ECOG performance status, primary tumor localization, metastatic lesion besides peritoneum, FL duration, preexisting diabetes, BMI, curative operation and regi- men type of SL (Table 2).

\section{Univariate and multivariate analysis for mOS in SL group}

Univariate analysis revealed ECOG performance status, peritoneal metastasis, number of metastasis, FL duration, thrombotic event, CA19-9 level above $90 \mathrm{U} / \mathrm{mL}$ and BMI were significant independent prognostic factors for $\mathrm{mOS}(\mathrm{p}<0.05)$. Therefore, the factors were included in the multivariate analysis.

In the multivariate analysis, more than one site of metastasis lesion, thrombotic event during FL and CA19-9 level above 90 $\mathrm{U} / \mathrm{mL}$ were significantly independent prognostic factors for mOS for all 245 patients in SL group ( $\mathrm{p}<0.05)$ (Table 3).

Table 2. Baseline Characteristics of Patients with mOS Less Than 6 Months and Greater Than 6 Months in the SL Group

\begin{tabular}{|c|c|c|c|}
\hline Characteristic & mOS 6 months below $(n=138)$ & mOS 6 months above $(n=107)$ & p-value \\
\hline Male sex & $65(47.1)$ & $55(51.4)$ & 0.522 \\
\hline Age, yr & $64.0(56.0-69.0)$ & $61.0(55.0-68.0)$ & 0.076 \\
\hline ECOG 0 & $110(79.7)$ & $95(88.8)$ & 0.145 \\
\hline Tumor localization & & & 0.451 \\
\hline Head & $68(49.3)$ & $60(56.1)$ & \\
\hline Body and tail & $60(43.5)$ & $38(35.5)$ & \\
\hline Overlapping & $10(7.2)$ & $9(8.4)$ & \\
\hline \multicolumn{4}{|l|}{ Metastatic lesion } \\
\hline Liver & $101(73.2)$ & $67(62.6)$ & 0.096 \\
\hline Lung & $26(18.8)$ & $24(22.4)$ & 0.525 \\
\hline Bone & $4(2.9)$ & $7(6.5)$ & 0.218 \\
\hline Other & $10(7.2)$ & $8(7.5)$ & 1.000 \\
\hline Distant lymph node & $29(21.0)$ & $21(19.6)$ & 0.873 \\
\hline Peritoneum & $60(43.5)$ & $20(18.7)$ & $<0.001$ \\
\hline No. of metastasis & & & $<0.001$ \\
\hline Single & $58(42.0)$ & $73(68.2)$ & \\
\hline Multiple & $80(58.0)$ & $34(31.8)$ & \\
\hline \multicolumn{4}{|l|}{ FL duration, wk } \\
\hline OS & $16.0(8.0-24.0)$ & $20.0(12.0-32.0)$ & 0.108 \\
\hline $\mathrm{mOS}$ & $12.0(8.0-24.0)$ & $36.0(28.0-52.0)$ & $<0.001$ \\
\hline Diabetes mellitus & $52(37.7)$ & $43(40.2)$ & 0.694 \\
\hline Thrombosis & $25(18.1)$ & $6(5.6)$ & 0.003 \\
\hline CA19-9 $\geq 90.0 \mathrm{U} / \mathrm{mL}$ & $98(71.0)$ & $59(55.1)$ & 0.011 \\
\hline BMI, kg/m² & $22.5(20.90-23.67)$ & $23.4(22.20-24.30)$ & 0.187 \\
\hline Curative operation & $36(26.1)$ & $28(26.2)$ & 0.989 \\
\hline Second line type & & & 0.217 \\
\hline FOLFIRINOX & $45(32.6)$ & $46(43.0)$ & \\
\hline FOLFOX & $15(10.9)$ & $13(12.1)$ & \\
\hline Capecitabine+0xaliplatin & $63(45.7)$ & 35 (32.7) & \\
\hline FEP & $15(10.9)$ & $13(12.1)$ & \\
\hline
\end{tabular}

Data are presented as number (\%) or median (interquartile range).

mOS, modified overall survival; SL, second-line chemotherapy; ECOG, Eastern Cooperative Oncology Group; FL, first-line chemotherapy; OS, overall survival; CA19-9, carbohydrate antigen 19-9; BMI, body mass index; FOLFIRINOX, 5-FU+folinic acid+irinotecan+oxaliplatin; FOLFOX, folinic acid+fluorouracil+oxaliplatin; FEP, folinic acid+etoposide+cisplatin. 
Table 3. Univariate and Multivariate Analyses of the mOS in the SL Group $(n=245)$

\begin{tabular}{|c|c|c|c|c|}
\hline & \multicolumn{2}{|c|}{ Univariate } & \multicolumn{2}{|c|}{ Multivariate } \\
\hline & HR (95\% CI) & $\mathrm{p}$-value & HR (95\% CI) & $\mathrm{p}$-value \\
\hline Sex & $1.059(0.822-1.363)$ & 0.659 & - & - \\
\hline Age & $1.011(0.997-1.026)$ & 0.121 & - & - \\
\hline \multicolumn{5}{|l|}{ ECOG } \\
\hline 0 & Reference & Reference & Reference & Reference \\
\hline$\geq 1$ & $0.400(0.164-0.978)$ & 0.044 & $1.364(0.933-1.992)$ & 0.109 \\
\hline Tumor localization & & & - & - \\
\hline Head & Reference & 0.057 & & \\
\hline Body and tail & $0.812(0.499-1.322)$ & 0.403 & & \\
\hline Overlapping & $1.128(0.690-1.845)$ & 0.631 & & \\
\hline Metastatic lesion & & & - & - \\
\hline Liver & $1.232(0.962-01.57)$ & 0.099 & & \\
\hline Lung & $1.024(0.764-1.372)$ & 0.876 & & \\
\hline Bone & $0.978(0.548-1.747)$ & 0.941 & & \\
\hline Other & $0.830(0.536-1.285)$ & 0.404 & & \\
\hline Distant lymph node & $0.994(0.744-1.328)$ & 0.967 & & \\
\hline Peritoneum & $1.622(1.236-2.127)$ & $<0.001$ & $1.253(0.924-1.698)$ & 0.147 \\
\hline \multicolumn{5}{|l|}{ No. of metastasis } \\
\hline Single & $0.621(0.481-0.802)$ & $<0.001$ & $0.694(0.520-0.926)$ & 0.013 \\
\hline Multiple & Reference & Reference & Reference & Reference \\
\hline \multicolumn{5}{|l|}{ SL type } \\
\hline FOLFIRINOX & Reference & Reference & Reference & Reference \\
\hline FOLFOX & $0.776(0.506-1.190)$ & 0.245 & $1.605(1.023-2.518)$ & 0.040 \\
\hline Capecitabine+Oxaliplatin & $1.151(0.680-1.949)$ & 0.601 & $1.710(1.233-2.372)$ & 0.001 \\
\hline FEP & $1.381(0.904-2.110)$ & 0.135 & $1.500(0.955-2.355)$ & 0.078 \\
\hline FL duration & $0.988(0.979-0.997)$ & 0.012 & $0.991(0.982-1.001)$ & 0.074 \\
\hline Diabetes mellitus & $0.830(0.655-1.051)$ & 0.123 & - & - \\
\hline Thrombosis & 1.789 (1.219-2.626) & 0.003 & $1.391(0.929-2.082)$ & 0.035 \\
\hline CA19-9 <90.0 U/mL & $0.662(0.504-0.868)$ & 0.003 & $1.184(0.881-1.591)$ & 0.025 \\
\hline CA19-9 $\geq 90.0 \mathrm{U} / \mathrm{mL}$ & Reference & Reference & Reference & Reference \\
\hline BMI & $0.926(0.861-0.995)$ & 0.037 & $0.940(0.875-1.010)$ & 0.090 \\
\hline Curative operation & $0.766(0.568-1.033)$ & 0.081 & - & - \\
\hline
\end{tabular}

mOS, modified overall survival; SL, second-line chemotherapy; HR, hazard ratio; CI, confidence interval; ECOG, Eastern Cooperative Oncology Group; FOLFIRINOX, 5-FU+folinic acid+irinotecan+oxaliplatin; FOLFOX, folinic acid+fluorouracil+oxaliplatin; FEP, folinic acid+etoposide+cisplatin; FL, first-line chemotherapy; CA19-9, carbohydrate antigen 19-9; BMI, body mass index.

\section{Prognostic nomogram and prediction of the survival ben- efit of SL}

The prognostic nomogram was devised with all statistically significant independent factors from the analysis of mOS groups divided by duration of 6 months (Fig. 1). Number of metastasis lesion, peritoneal metastasis, thrombotic event during FL and CA19-9 were the final determinants integrated to the nomogram. The prognostic score was based on the total number of points obtained from the nomogram.

\section{Confirmation of the prediction model with prognostic subgroups}

We identified three prognostic subgroups with the nomogram scoring: "good" prognosis (score 0-60), "intermediate" prognosis (score 116-160) and "poor" prognosis (score 176-276). Good prognosis group included: none of the four determinants (score 0 ), only peritoneal metastasis (score 58), only single metastasis (score 58), only CA19-9 above $90 \mathrm{U} / \mathrm{mL}$ (score 60). Poor prognosis group included any three determinants together (scores 176 and 218) or all four significant factors included (maximum 


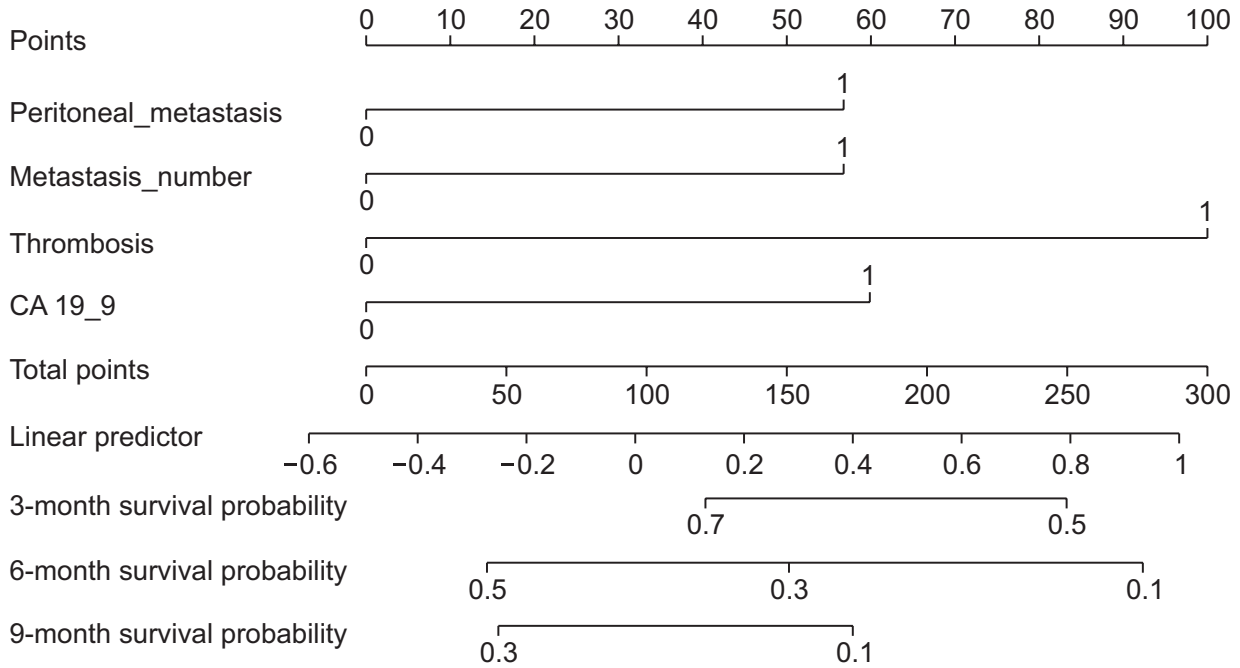

Fig. 1. Prognostic nomogram to predict individual modified overall survival (mOS) probability. Prognostic nomogram to predict individual m0S probability at the beginning of second-line chemotherapy in patients with advanced pancreatic cancer. First, the points associated with each of the four prognostic factors are obtained via upward vertical translation of the patient's variable value to the line labeled "Points." Next, the points are summed and the corresponding total number is reported as a dot on the line labeled "Total points." A vertical line is then drawn downward from the total point dot to obtain the mOS prediction at the intersection with the "3-," "6-,"and "9-month survival probability" lines.

No peritoneal metastasis: 0 , peritoneal metastasis: 1; single metastatic lesion: 0 , multiple metastatic lesions: 1 ; No thrombotic event: 0 , thrombotic event: 1; carbohydrate antigen 19-9 (CA19-9) <90 U/mL: 0, CA19-9 $\geq 90$ U/mL: 1.

score 276). The expected survival after the FL failure represented as median mOS was 28.0 weeks (95\% CI, 25.2 to 30.8) in good prognosis group, 18.0 weeks (95\% CI, 15.8 to 210.2) in intermediate prognosis group, and 12.0 weeks (95\% CI, 10.7 to 13.3) in poor prognosis group $(\mathrm{p}<0.001)$. Such grouping achieved a clear separation of the Kaplan-Meier curves (Fig. 2).

\section{Internal validation and external validation of the final nomogram}

The discrimination ability of the final scoring model developed in the main analysis was internally confirmed: the Harrell's C-index was 0.620 and the iAUC curve of the development cohort was 0.619. Data of the four baseline parameters that were required for the nomogram calculation was available for 123 patients from the external validation cohort: the Harrell's Cindex was 0.560 and the iAUC curve of the external validation cohort was 0.562 (Fig. 3).

\section{DISCUSSION}

Generally, pancreatic cancer progression is very rapid and patient's performance status falls even more rapidly not enough to be suitable for any salvage chemotherapy when the FL is turned out to be ineffective..$^{10,11}$ Due to the aggressive manner of the disease and the toxicity of chemotherapy, consideration for SL should be more cautious of the risk and benefit. ${ }^{12}$

Current guideline for advanced pancreatic cancer recommends SL after the FL failure, even though the benefit of SL remains to be controversial. ${ }^{13}$ The CONKO-003 study, an open

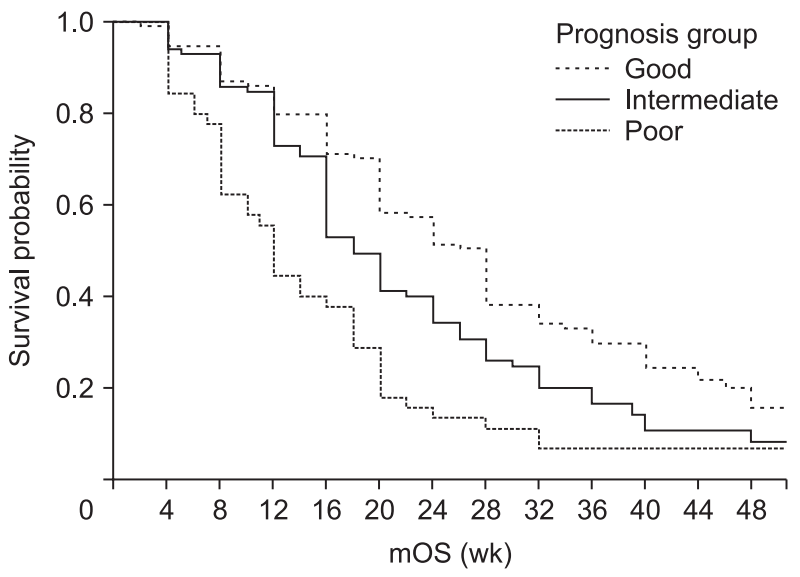

Fig. 2. Kaplan-Meier curves of modified overall survival (mOS) for the prognostic subgroups "good-intermediate-poor" in the development cohort.

randomized phase 3 study, proved that the combination of oxaliplatin, 5-FU, and folinic acid (OFF regimen) after previous gemcitabine-based chemotherapy was superior to BSC in terms of PFS (13 weeks vs 9 weeks) and OS (26 weeks vs 13 weeks). ${ }^{7}$ However, there is no more concrete evidence to support the usefulness of SL for gemcitabine-refractory advanced pancreatic cancer. $^{1,13}$

Considering that the clinical benefit of currently available SL is marginal, it is very important to find the clinical parameters for determining the potential benefit of the salvage treatment when the patients are enfaced the failure of FL. ${ }^{14-16}$ Maréchal et al. ${ }^{17}$ reported that patient's performance status and initial albu- 

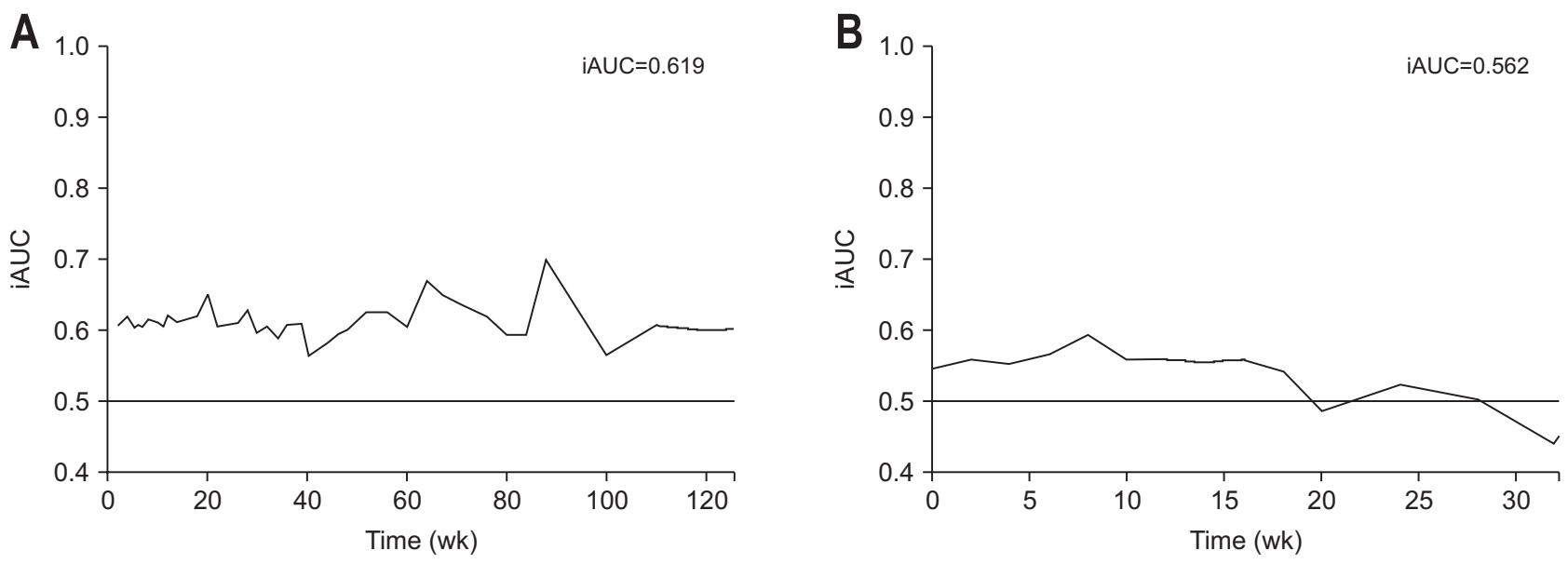

Fig. 3. Internal validation iAUC (A) and external validation iAUC (B) of the prognostic nomogram. iAUC, incremental area under the curve.

min level were independent prognostic factors in chemotherapy naive and gemcitabine-refractory patients with advanced pancreatic cancer. Petrelli et al. ${ }^{18}$ suggested the responsiveness to the FL with gemcitabine was predictive factor for SL with OFF in advanced pancreatic cancer. CA19-9 also was suggested as one of the important prognostic markers for SL. ${ }^{7,21}$

In this study, we tried to develop a simplified and useful prognostic model that could clinically predict which patients can have the benefit of SL from the retrospective analysis of single center cohort data. Our study identified prognostic factors influencing the survival of advanced pancreatic cancer patients after the progression of FL. The significant factors depending on the mOS were peritoneal metastasis, number of metastatic lesions, CA19-9 level and thrombotic event. Based on these findings, we made a nomogram scoring system. Despite the fact that the Harrell's S-index and iAUC internal validation index of the nomogram were not so powerful, this scoring system allows reliable differentiation into three prognostic groups (good, intermediate, and poor) which demonstrate clear differences in mOS. Patients in the poor prognosis group had a median mOS of about 12 weeks, and those in the good prognosis group of about 28 weeks, demonstrating a difference of about 16 months.

Despite the fact that pragmatic parameters are already frequently used in decision making for the initiation of SL, our final nomogram presents simplicity compared to previous approach with multiple and complex variables of the scoring system. In addition to helping the decision-making process for clinicians, this nomogram may be beneficial for selecting patients for SL. Moreover, with this prognostic monogram, the development of risk adapted therapies for pancreatic cancer in palliative SL management could be also considered in the different risk groups identified by the scoring system.

There are several points which provide differentiation among other similar studies. The inclusion period in our study was long enough, from 2010 to 2015, to include sen- sational regimens introduced in the field. This time period is very important in the era of pancreatic cancer since clinical practices have changed with the approval of the 5-FU+folinic acid+irinotecan+oxaliplatin (FOLFIRINOX) and gemcitabine plus nab-paclitaxel chemotherapy. ${ }^{22}$ Our study evaluated a large cohort population including patients treated with original or modified FOLFIRINOX regimen in SL, which was approved in 2011 and widely available from 2014. ${ }^{23}$ Parameters used in the model are clinically approached, simple to collect for clinicians, and consistent with the previous models devised. Internal validation was demonstrated to prove the satisfactory performance and validity of the nomogram. Our prognostic nomogram was externally validated through patient group in 2016 of Yonsei University Hospital. Therefore, our final prognostic nomogram made an attempt to confirm the reliability in the era of new, highly efficient treatment regimens for advanced pancreatic cancer. Few similar prognostic models suggested currently have limitation of heterogeneity in FL. However, our study made assess to similar prognostic factors in homogenous gemcitabinebased FL patient groups. ${ }^{24}$

However, there are limitations in generalizing our prognostic nomogram due to the retrospective nature of the study. Due to the slightly low C-index of the nomogram, the predictive ability of the scoring system is not fully qualified to be used in general practice yet. Moreover, the external validation also showed low predictive ability. Hopefully, with the significant variables from the analysis, the predictive ability will improve with more data sets. External validation in different hospitals and in other countries will definitively confirm the worldwide relevance of the model in the future. Thus, the prognostic usefulness of our nomogram needs to be confirmed through a large prospective validation in the future.

Many oncologic approaches are currently made to figure out which patients truly benefit from receiving SL on molecular basis but little is being researched on clinical basis. ${ }^{25}$ There is 
no worldwide consensus of clinical parameter scoring system available to support the physician's decision to go on with SL or give up palliative chemotherapy and start BSC. ${ }^{24}$ Despite the limitations mentioned above, this study with our prognostic nomogram based scoring system may help to develop strategies to identify patients who can obtain real benefit from SL after progression of gemcitabine-based FL.

\section{CONFLICTS OF INTEREST}

No potential conflict of interest relevant to this article was reported.

\section{AUTHOR CONTRIBUTIONS}

Study concept and design: S.B., J.E.L. Data acquisition: J.E.L. Data analysis and interpretation: J.E.L. Drafting of the manuscript: S.B., J.E.L. Critical revision of the manuscript for important intellectual content: H.S.L., M.J.C., J.Y.P., S.W.P., S.Y.S. Statistical analysis: J.E.L. Administrative, technical, or material support; study supervision: S.B., H.S.L.

\section{ORCID}

Jeung Eun Lee

Hee Seung Lee

Moon Jae Chung

Jeong Youp Park

Seung Woo Park

Si Young Song

Seungmin Bang https://orcid.org/0000-0002-1658-3322 https://orcid.org/0000-0002-2825-3160 https://orcid.org/0000-0002-5920-8549 https://orcid.org/0000-0003-0110-8606 https://orcid.org/0000-0001-8230-964X https://orcid.org/0000-0002-1417-4314 https://orcid.org/0000-0001-5209-8351

\section{REFERENCES}

1. Oettle H, Riess H, Stieler JM, et al. Second-line oxaliplatin, folinic acid, and fluorouracil versus folinic acid and fluorouracil alone for gemcitabine-refractory pancreatic cancer: outcomes from the CONKO-003 trial. J Clin Oncol 2014;32:2423-2429.

2. Rahma OE, Duffy A, Liewehr DJ, Steinberg SM, Greten TF. Second-line treatment in advanced pancreatic cancer: a comprehensive analysis of published clinical trials. Ann Oncol 2013;24:19721979.

3. Passero FC Jr, Saif MW. Second line treatment options for pancreatic cancer. Expert Opin Pharmacother 2017;18:1607-1617.

4. Gill S, Ko YJ, Cripps C, et al. PANCREOX: a randomized phase III study of fluorouracil/leucovorin with or without oxaliplatin for second-line advanced pancreatic cancer in patients who have received gemcitabine-based chemotherapy. J Clin Oncol 2016;34:3914-3920.

5. Sarabi M, Mais L, Oussaid N, Desseigne F, Guibert P, De La Fouchardiere C. Use of gemcitabine as a second-line treatment following chemotherapy with FOLFIRINOX for metastatic pancreatic adenocarcinoma. Oncol Lett 2017;13:4917-4924.

6. Rahman FAU, Ali S, Saif MW. Update on the role of nanoliposomal irinotecan in the treatment of metastatic pancreatic cancer. Therap Adv Gastroenterol 2017;10:563-572.

7. Sinn M, Dälken L, Striefler JK, et al. Second-Line treatment in pancreatic cancer patients: who profits? Results from the CONKO study group. Pancreas 2016;45:601-605.

8. Aprile G, Negri FV, Giuliani F, et al. Second-line chemotherapy for advanced pancreatic cancer: which is the best option? Crit Rev Oncol Hematol 2017;115:1-12.

9. Sonbol MB, Firwana B, Wang Z, et al. Second-line treatment in patients with pancreatic ductal adenocarcinoma: a meta-analysis. Cancer 2017;123:4680-4686.

10. Fiore M, Ramella S, Valeri S, et al. Phase II study of induction chemotherapy followed by chemoradiotherapy in patients with borderline resectable and unresectable locally advanced pancreatic cancer. Sci Rep 2017;7:45845.

11. Vernerey D, Huguet F, Vienot A, et al. Prognostic nomogram and score to predict overall survival in locally advanced untreated pancreatic cancer (PROLAP). Br J Cancer 2016;115:281-289.

12. Kim ST, Choi YJ, Park KH, et al. A prognostic model to identify patients with advanced pancreas adenocarcinoma who could benefit from second-line chemotherapy. Clin Oncol (R Coll Radiol) 2012;24:105-111.

13. Chung KH, Ryu JK, Son JH, et al. Efficacy of capecitabine plus oxaliplatin combination chemotherapy for advanced pancreatic cancer after failure of first-line gemcitabine-based therapy. Gut Liver 2017;11:298-305.

14. Custodio A, Puente J, Sastre J, Díaz-Rubio E. Second-line therapy for advanced pancreatic cancer: a review of the literature and future directions. Cancer Treat Rev 2009;35:676-684.

15. Boeck S, Heinemann V. Second-line therapy in gemcitabinepretreated patients with advanced pancreatic cancer. J Clin Oncol 2008;26:1178-1179.

16. Berger AK, Abel U, Komander C, Harig S, Jäger D, Springfeld C. Chemotherapy for advanced pancreatic adenocarcinoma in elderly patients ( $\geq 70$ years of age): a retrospective cohort study at the National Center for Tumor Diseases Heidelberg. Pancreatology 2014;14:211-215.

17. Maréchal R, Demols A, Gay F, et al. Prognostic factors and prognostic index for chemonaïve and gemcitabine-refractory patients with advanced pancreatic cancer. Oncology 2007;73:41-51.

18. Petrelli F, Inno A, Ghidini A, et al. Second line with oxaliplatinor irinotecan-based chemotherapy for gemcitabine-pretreated pancreatic cancer: a systematic review. Eur J Cancer 2017;81:174182.

19. Fornaro L, Vivaldi C, Cereda S, et al. Second-line chemotherapy in advanced biliary cancer progressed to first-line platinum-gemcitabine combination: a multicenter survey and pooled analysis with published data. J Exp Clin Cancer Res 2015;34:156.

20. Viaud J, Brac C, Artru P, et al. Gemcitabine as second-line chemotherapy after FOLFIRINOX failure in advanced pancreatic adeno- 
carcinoma: a retrospective study. Dig Liver Dis 2017;49:692-696.

21. Ouyang H, Ma W, Liu F, et al. Factors influencing survival of patients with pancreatic adenocarcinoma and synchronous liver metastases receiving palliative care. Pancreatology 2017;17:773781.

22. Gharaibeh M, McBride A, Bootman JL, Patel H, Abraham I. Economic evaluation for the US of nab-paclitaxel plus gemcitabine versus FOLFIRINOX versus gemcitabine in the treatment of metastatic pancreas cancer. J Med Econ 2017;20:345-352.

23. Kobayashi N, Shimamura T, Tokuhisa M, Goto A, Endo I, Ichikawa
Y. Effect of FOLFIRINOX as second-line chemotherapy for metastatic pancreatic cancer after gemcitabine-based chemotherapy failure. Medicine (Baltimore) 2017;96:e6769.

24. Vienot A, Beinse G, Louvet C, et al. Overall survival prediction and usefulness of second-line chemotherapy in advanced pancreatic adenocarcinoma. J Natl Cancer Inst 2017;109.

25. Burris HA 3rd, Moore MJ, Andersen J, et al. Improvements in survival and clinical benefit with gemcitabine as first-line therapy for patients with advanced pancreas cancer: a randomized trial. J Clin Oncol 1997;15:2403-2413. 